\title{
Ionization of water as an effect of quantum delocalization at aqueous electrode interfaces
}

\author{
Jinggang Lan, ${ }^{*}$ Vladimir V. Rybkin, and Marcella lannuzzi* \\ Department of Chemistry, University of Zurich, Switzerland \\ E-mail: jinggang.lan@chem.uzh.ch; marcella.iannuzzi@chem.uzh.ch \\ Phone: +41446354479
}

\begin{abstract}
The enhanced probability of water dissociation at the aqueous electrode interfaces is predicted by path-integral ab initio molecular dynamics. The ionization process is observed at the aqueous platinum interface when nuclear quantum effects are introduced in the statistical sampling, while minor effects have been observed at the gold interface. We characterize the dissociation mechanism and the dynamics of the formed water ions. In spite of the fact that the concentration and lifetime of the ions might be challenging to be experimentally detectable, they may serve as a guide to future experiments. Our observation might have a significant impact on the understanding of electrochemical processes occurring at the metal electrode surface.
\end{abstract}

Understanding the aqueous solid interfaces is of great interest to electrochemistry. Many important processes in electrochemical reactions occur within the electrical double layers (EDLs). However, the direct observation and characterization of the interfaces under operating conditions are challenging for both experimental and modeling approaches. ${ }^{1-5}$ Important aspects to take into consideration are the reactivity and dissociation of water near the metal surface, which might affect other electrochemical reactions. ${ }^{6-8}$ The water's wetting layer on 
metals has been extensively studied under ultra-high vacuum conditions using vibrational spectroscopy and density functional theory calculations, ${ }^{6,9-12}$ focusing in particular on the hydrogen-bond network. Another discussed issue is the local proton disorder within the wetting bilayer, as induced by water dissociation, proton transfer, and formation of $\mathrm{H}_{3} \mathrm{O}-$ and $\mathrm{OH}$-like surface species. ${ }^{9,13}$ Temperature-programmed desorption experiments seem to confirm that water on $\mathrm{Pt}(111)$ is forming a "hydronium-hydroxide" hydrate, ${ }^{14}$ though these findings are still controversial. ${ }^{15}$ Even more challenging is the characterization of the water/metal interface within a complex heterogeneous system under ambient conditions. Recent advances in surface sensitive spectroscopy such as X-ray absorption spectroscopy, in situ Raman spectroscopy, as well as quantum dynamics methods are expected to be able to resolve structural transitions of interfacial water. ${ }^{3,4,8,16}$

The reversible formation of adsorbed $\mathrm{OH}\left(\mathrm{OH}_{\mathrm{ad}}\right)$ on $\mathrm{Pt}(111)\left(\mathrm{Pt}+\mathrm{H}_{2} \mathrm{O} \rightleftharpoons \mathrm{Pt} \ldots \mathrm{OH}+\mathrm{H}^{+}+\mathrm{e}^{-}\right)$ has been observed at surface potentials stronger than $0.55 \mathrm{~V}$ versus the reversible hydrogen electrode. ${ }^{8,17,18}$ At potentials lower than $0.35 \mathrm{~V}$, hydrogen is known to adsorb on $\mathrm{Pt}(111)$ : $\mathrm{H}_{3} \mathrm{O}^{+}+\mathrm{e}^{-}+\mathrm{Pt} \rightleftharpoons \mathrm{Pt} . . \mathrm{H}+\mathrm{H}_{2} \mathrm{O}$. At intermediate potentials, between $0.35-0.55 \mathrm{~V}$, water is generally believed not to react with $\mathrm{Pt}(111)$. Under these conditions, the current flowing through the "double-layer region" is associated to the capacitive charging of the EDLs. ${ }^{19,20}$ Better understanding and control on the nature of dissociative processes within the EDL is then desirable, since these have a significant impact on the concentration of reactive species at the interface.

From the theoretical side, ab initio molecular dynamics (AIMD) makes it feasible to model realistic electrochemical interfaces, albeit often at significant computational cost. ${ }^{1-5,16,21-24}$ Recently, AIMD has been applied to determine and understand the potential of zero charge (PZC) of metal-water interfaces ${ }^{21,24}$ and the structure of the EDL of electrified interfacial water. ${ }^{2}$ In our previous work, we used AIMD to elucidate the experimentally observed effects of $\mathrm{CO}$ coverage and water co-adsorption on the CO-stretching mode. ${ }^{22}$

The commonly used semi-classical AIMD approach, however, neglects possible nuclear 
quantum effects (NQEs), and this might lead to an insufficient statistical sampling of the dynamics of light atoms. ${ }^{25,26}$ When water dissociation and hydrogen/proton dynamics are involved, including NQEs may disclose otherwise not accessible pathways. ${ }^{26-31}$ For instance, NQEs favour hydrogen delocalization within the water-hydroxyl overlayer at transition metal surfaces, ${ }^{27}$ and water-monomer dissociation on $\mathrm{Pt}(211) .{ }^{29}$ Indeed, experiments with deutereted water have revealed kinetic isotope effects in electrochemical systems, while quantum tunneling is known to be responsible for the electron-proton-driven transformation of dioxygen at low overpotential. ${ }^{32}$ Nevertheless, NQEs at aqueous solid interfaces are rarely considered in simulations. ${ }^{33}$
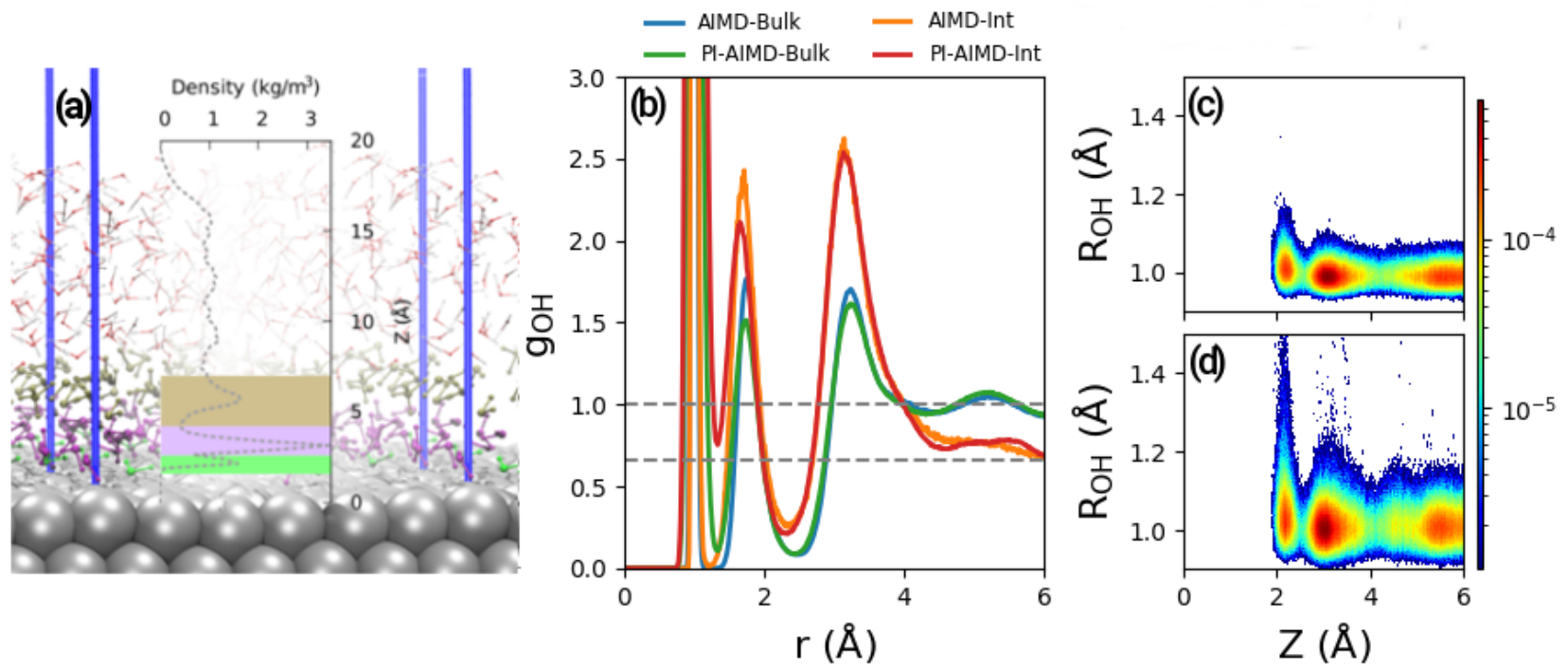

Figure 1: (a) Ball and stick representation of the model consisting of the Pt(111) slab in contact with liquid water. The blue lines delimit the simulation cell in periodic boundary conditions. Color code: silver spheres are $\mathrm{Pt}$ atoms, red $\mathrm{O}$ bulk atoms, grey $\mathrm{H}$ bulk atoms, while the molecules belonging to the first three layers are coloured in green, purple and olive. Inset: density of water as a function of $\mathrm{Z}$ coordinate, where $\mathrm{Z}=0$ is set at the topmost $\mathrm{Pt}$ layer. The shaded areas correspond to the first three water layers. (b) $\mathrm{OH}$ radial distribution function computed separately for the $\mathrm{O}$ atoms at the interface and in bulk (gOH at interface normalized to $\frac{2}{3}$ due to the reduced number of degrees of freedom in the confined water layers). (c) Distribution of the intra-molecular $\mathrm{OH}$ bond length as obtained from AIMD and (d) from PI-AIMD. Distributions' color code: from blue to red indicates an increased probability of finding the respective value. The distributions are obtained as averages over all $\mathrm{OH}$, all PI-beads, and the whole trajectories.

In this work we compare extensive AIMD and path-integral (PI) AIMD simulations to 
determine the role of NQEs on the structure and dynamics of liquid water in contact with the $\mathrm{Pt}(111)$ and $\mathrm{Au}(111)$ surfaces. In particular, we show that water molecules adsorbed at atop $\mathrm{Pt}$ sites have a higher dissociation rate into $\mathrm{OH} / \mathrm{H}_{3} \mathrm{O}$ like pairs than on $\mathrm{Au}$ or in bulk liquid. However, this behaviour is observed only by PI-AIMD, which provides unambiguous evidence for water ionization via quantum delocalisation. Moreover, by quantifying the statistical probability of the dissociation events, since the $\mathrm{OH}$ forms a hydroxide with the metal, the finite concentration of solvated $\mathrm{H}^{+}$determines an excess of positive charge at the interface, without applying any bias.

All MD simulations are performed within the canonical ensemble at $300 \mathrm{~K}$. The AIMD trajectories are generated using the second-generation Car-Parrinello molecular dynamics scheme. ${ }^{34}$ The PI-AIMD sampling is obtained in the framework of the generalized Langevin equation thermostat (PIGLET) method, ${ }^{28}$ as implemented in I-PI, ${ }^{35}$ using six beads per atom. The interface model consists of a $(6 \times 6)$ four-layer metallic slab in contact with a 15 Å-thick water film (see Fig. 1(a)).

The characteristic density profile of water in contact with $\operatorname{Pt}(111)$ obtained with the two sampling procedures is equivalent (see inset of Fig. 1(a)). The first structured layer is found at $2.2 \AA$ (green region), the second maximum at 3.1 (purple region) and the third at $5.7 \AA$ (olive region). This latter separates the contact double layer from the water bulk region. The resulting profile is in pretty good agreement with experiments, which estimate a thickness of the interface region between 3 and $5 \AA .{ }^{36}$ The water molecules within the first layer are chemisorbed, with the $\mathrm{O}$ atom on top of a surface Pt atom. The angle between the $\mathrm{OH}$ bonds of the adsorbed molecules and the surface normal (See Fig.S1) is confined between $60^{\circ}$ and $80^{\circ}$, as shown by the cosine distribution in Fig. S8. Hence, these $\mathrm{OH}$ bonds are either parallel to the surface or form a H-bond with a molecule of the second layer. Within first two layer of water, there are about 29 water molecules and 6 of them lay on the first chemisorb layer, resulting a coverage of $1 / 6$. The OH's of the molecules belonging to the second layer are mainly pointing towards the surface. The molecules within the third 
layer, instead, direct the OH's towards the bulk, whereas within the bulk region they are, as expected, isotropic oriented. This means that hydrogen bonding between the contact layer and the bulk is rare. This observation is consistent with the work by Limmer et al., as well as with our previous study on water and $\mathrm{CO}$ co-adsorption, ${ }^{22}$ where it is shown that water exchange at the hydrated platinum electrode is rare. ${ }^{37}$

While the NQEs seem not to affect the density and orientation of the adsorbed molecules, they play a role in the sampling of bond distances, as evinced from the $\mathrm{O}-\mathrm{H}$ radial distribution function $g_{\mathrm{OH}}$. In Fig. 1(b) we compare the $g_{\mathrm{OH}}$ obtained from bulk and interfacial water, with and without NQEs. The quantum water-bulk $g_{\mathrm{OH}}$ differs from the classical one only for the broadening of the first peak, corresponding to the intramolecular $\mathrm{OH}$ bond. At the interface, instead, the quantum $g_{\mathrm{OH}}$ (red in Fig. 1(b)) shows a significant probability of finding the $\mathrm{H}$ atom at distances between 1.2 and $1.5 \AA$, i.e., longer than a $\mathrm{OH}$ bond and shorter than a hydrogen bond. These configurations are associated to proton transfer events. The resulting $\mathrm{OH}_{3}^{+}$forms shorter hydrogen bonds with the neighboring oxygens, leading to the broadening towards shorter distances of the second $g_{\mathrm{OH}}$ peak. The probability distribution of the shortest $\mathrm{OH}$ distances, $R_{\mathrm{OH}}$ (taking for each $\mathrm{O}$ the two closest $\mathrm{H}$ atoms), as a function of the height of the oxygen over the metal's surface $(Z)$ is reported in the panels (c) and (d) of the same figure, for the classical and the quantum trajectory, respectively. The quantum distribution presents a slight elongation of the $\mathrm{OH}$ also in the bulk $(\mathrm{Z}>5 \AA)$, as already suggested by the broadening of the first $g_{\mathrm{OH}}$ peak. However, the most striking changes are associated with the molecules within the interface double layer. Here, the enhanced delocalization of the hydrogens results in $\mathrm{O}-\mathrm{H}$ distances characteristic of hydrogen bonds, pointing to ionization events.

The observed self-ionization processes at the interface are fast and involve more molecules, among which one is adsorbed at a metal-top site (See Movie.S1 in Supplementary Information). For instance, the snapshots illustrated in Fig. 2 are taken every 10 fs along a trajectory's segment during which one of such events occurs. The process starts by the de- 


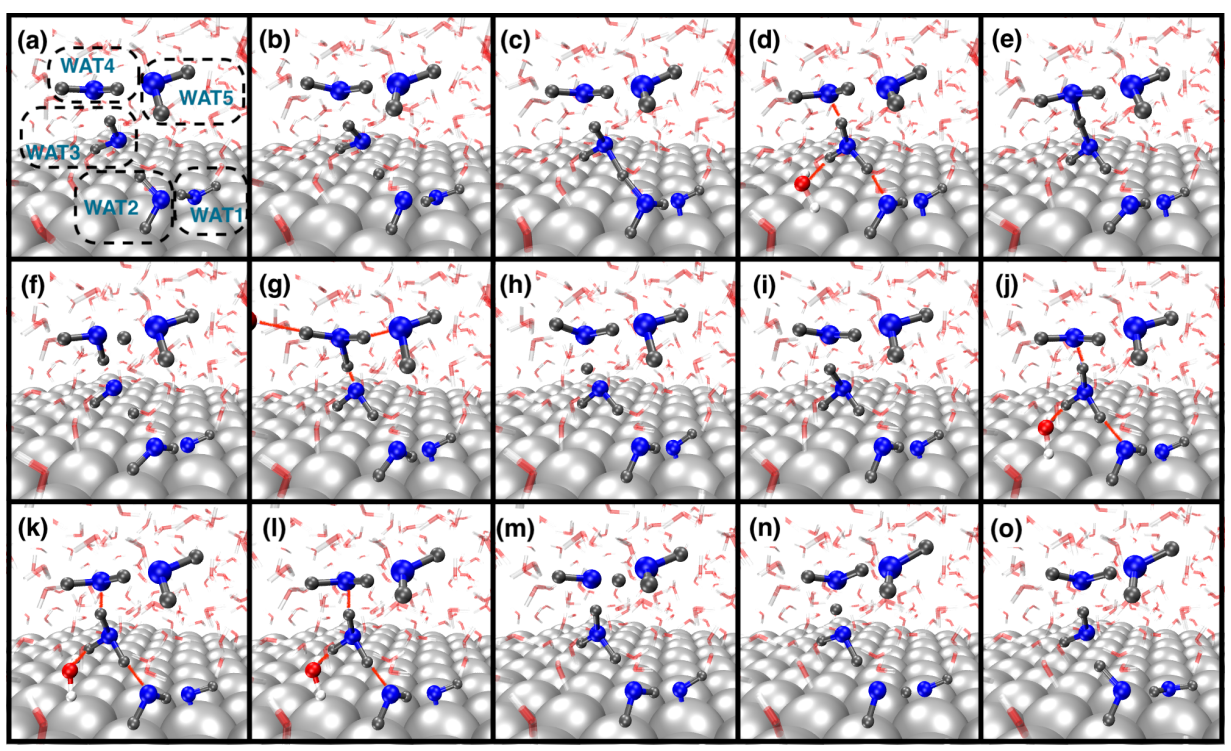

Figure 2: Sequence of snapshots extracted from the PI-AIMD trajectory during one selfionization event. The frames cover a time of $150 \mathrm{fs}$ and are taken every $10 \mathrm{fs}$. The starting point after 5 ps of simulation time. The water molecules involved in the process are in the ball-stick representation (blue and grey), while the line representation (red and white) is used for the surrounding liquid. For those uncoordinated protons can be regarded as transition states

protonation of the adsorbed molecule, which becomes an adsorbed hydroxyl $\left(\mathrm{OH}^{*}\right)$ strongly bound to the surface (panel c). The proton is transferred to the hydrogen-bonded molecule. The dynamics evolves by a series of hydrogen hops along the hydrogen bond network connecting the first adsorbed molecule to other molecules in the contact bi-layer. This gives rise to the alternate formation of Zundel-like species $\left(\mathrm{H}_{5} \mathrm{O}_{2}^{+}\right)$, where the proton is shared by two water molecules (panel c, e, f, h, n), and Eigen-like species $\left(\mathrm{H}_{9} \mathrm{O}_{4}^{+}\right)$, where the hydronium ion coordinates three other molecules (panel d, g, i, j, k, l, m). In the illustrated case, the Pt-OH finally recombines to a proton after 120 fs. For all events occurring along the here discussed PI-AIMD trajectory, the dissociated proton remains confined within the contact double layer, with an average life time of about $200 \mathrm{fs}$.

The structural parameter proposed by Hassanali et al., ${ }^{38} N_{\text {ion }}$, is well suited for the statistical analysis on the presence and life time of separated protons and hydroxyl groups. Its analytical expression is given in Eq. 4. It tracks the oxygens with number of bonded $\mathrm{H}$ 
atoms different from 2 , given a bonding radius (we use $r_{0}=1.32 \AA$ ). By this definition, $N_{\text {ion }}$ is nearly zero when all water molecules are intact, it becomes $\sim 2$ when one $\mathrm{OH}-\mathrm{H}_{3} \mathrm{O}$ pair is formed, and it increases to larger values if more molecules dissociate. It is worth noting that by choosing a cutoff of $1.32 \AA$, this parameter specifically addresses the bonded $\mathrm{H}$ and not the hydrogen bonds.

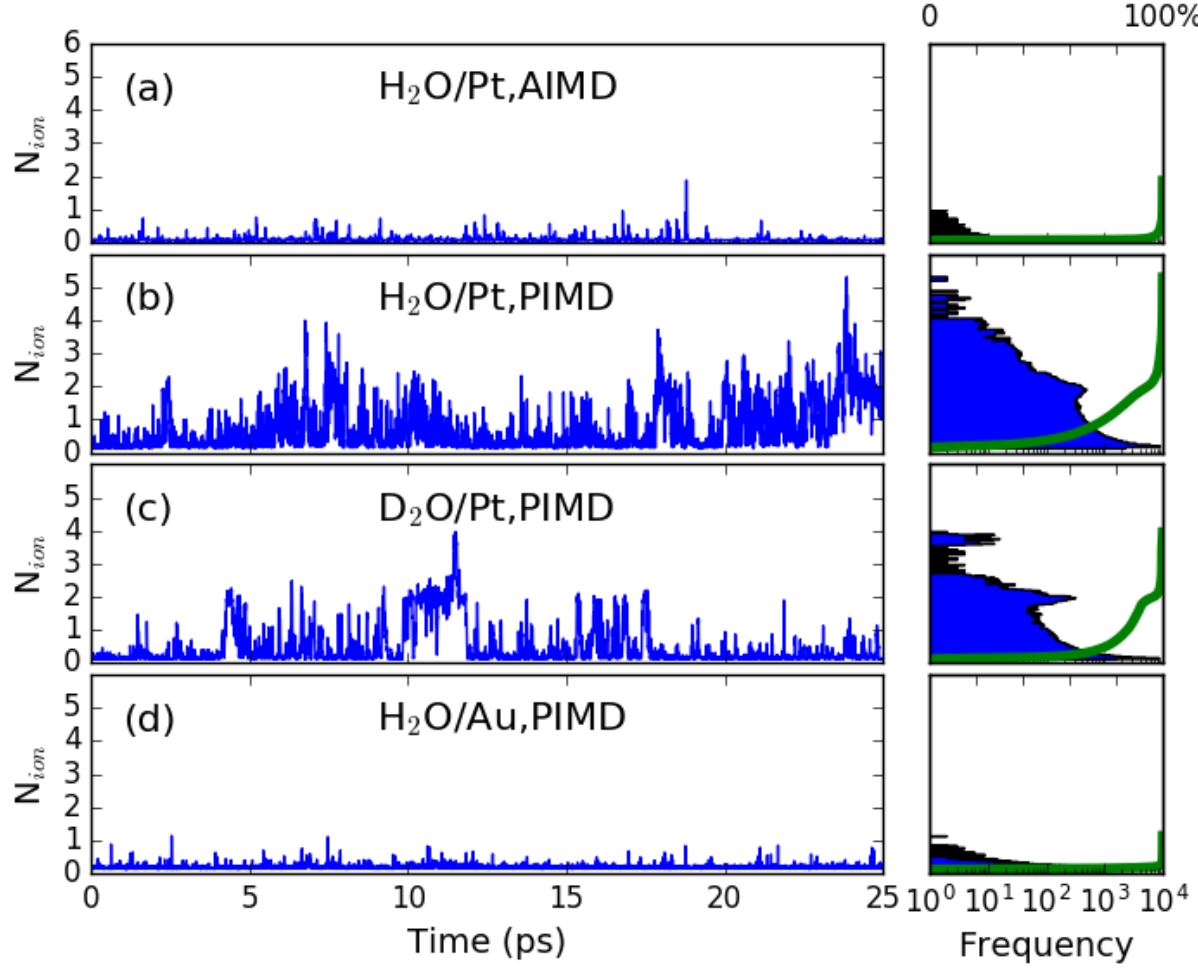

Figure 3: $N_{\text {ion }}$ calculated at each MD step for classical $\mathrm{H}_{2} \mathrm{O} / \mathrm{Pt}$ (a) and quantum $\mathrm{H}_{2} \mathrm{O} / \mathrm{Pt}$ (b), and for quantum $\mathrm{D}_{2} \mathrm{O} / \mathrm{Pt}(\mathrm{c})$ and $\mathrm{H}_{2} \mathrm{O} / \mathrm{Au}(\mathrm{d})$. The right panels are the corresponding effective counting of observations for each $N_{\text {ion }}$ value, plotted in logarithmic scale. The green curve measures the cumulative percentage of the probability distribution.

$N_{\text {ion }}$ recorded along the classical trajectory is always close to zero, as shown in Fig. 3(a), reflecting the fact that all molecules are intact and the respective $\mathrm{OH}$ bonds fluctuate $\pm 0.2 \AA$ around their equilibrium length. This is confirmed by the probability distribution (Fig. 3(a) right panel) showing that nearly $100 \%$ of configurations corresponds to $N_{\text {ion }}=0$. Along the quantum trajectory, instead, the variations of $N_{\text {ion }}$ are significantly larger, due to the enhanced amplitude of the OHs' length fluctuation all over the water slab. Moreover, we 
specifically identify ionization events whenever $N_{\text {ion }}$ increases above 2 . According to the probability distribution plotted in the right panel of Fig. 3(b), solvated protons, and corresponding $\mathrm{OH}^{*}$, are present in about $7 \%$ of the sampled configurations (from $\frac{\text { Freq }\left(\mathrm{N}_{\text {ion }} \geqslant 2\right)}{\mathrm{N}_{\text {samples }}}$ ). The coverage of $\mathrm{OH}^{*}$ at equilibrium is about $2 \times 10^{-5}$. Performing the same analysis for heavy water (see Fig. 3(c)), it turns out that ionized molecules are present only $3.5 \%$ of the time. No ionization events have been observed, instead, along the quantum trajectory of water in contact with the Au surface (see Fig. 3(c)).

The probability distribution of the oxygen coordination $\left(\mathrm{CN}_{\mathrm{O}}\right.$ in Eq. 4) mapped as a function of the O's position over Pt in Fig. 4(a) confirms that the dissociated $\mathrm{OH}$ is bound to the surface $(Z<2.5 \AA)$, while the solvated proton remains confined within the contact bi-layer $(2.5<Z<4)$. Nevertheless, the large majority of sampled molecular geometries are intact $\left(\mathrm{CN}_{\mathrm{O}}=2\right)$. This is the only encountered configuration in bulk (histogram in Fig. 4(b)) and at the liquid/vacuum interface. At the Pt interface the probability of finding a dissociated molecule is not negligible, but small. More precisely, it is three orders of magnitude smaller with respect to intact molecules, as shown by the $\mathrm{CN}_{O}$ histogram in Fig. 4(c) (the statistics is obtained over the whole trajectory).

In bulk water, the spontaneous fluctuations of the local electric field associated to the molecular motions are known to be able to induce self ionization processes. ${ }^{39}$ In particular, the AIMD study by Saitta et al. shows that electric fields beyond a threshold of about 0.35 $\mathrm{V} / \AA$ might trigger the dissociation thus generating an ionic current via a series of correlated proton jumps. ${ }^{40}$ Water close to the platinum surface has higher density compared to bulk water and forms a seemingly ordered phase. Such ordered water is generally expected to exhibit smaller dielectric constant because of surface-induced alignment of the molecular dipoles. ${ }^{41}$ The resulting electric field is expected to be more pronounced than the one generated by the random fluctuations in the bulk, as also inferred from the profile of the electrostatic potential in Fig.S2. This explains the elongation of the $\mathrm{OH}$ bonds at the interface, which has been observed also along the classical trajectory. The introduction of NQEs systematically 

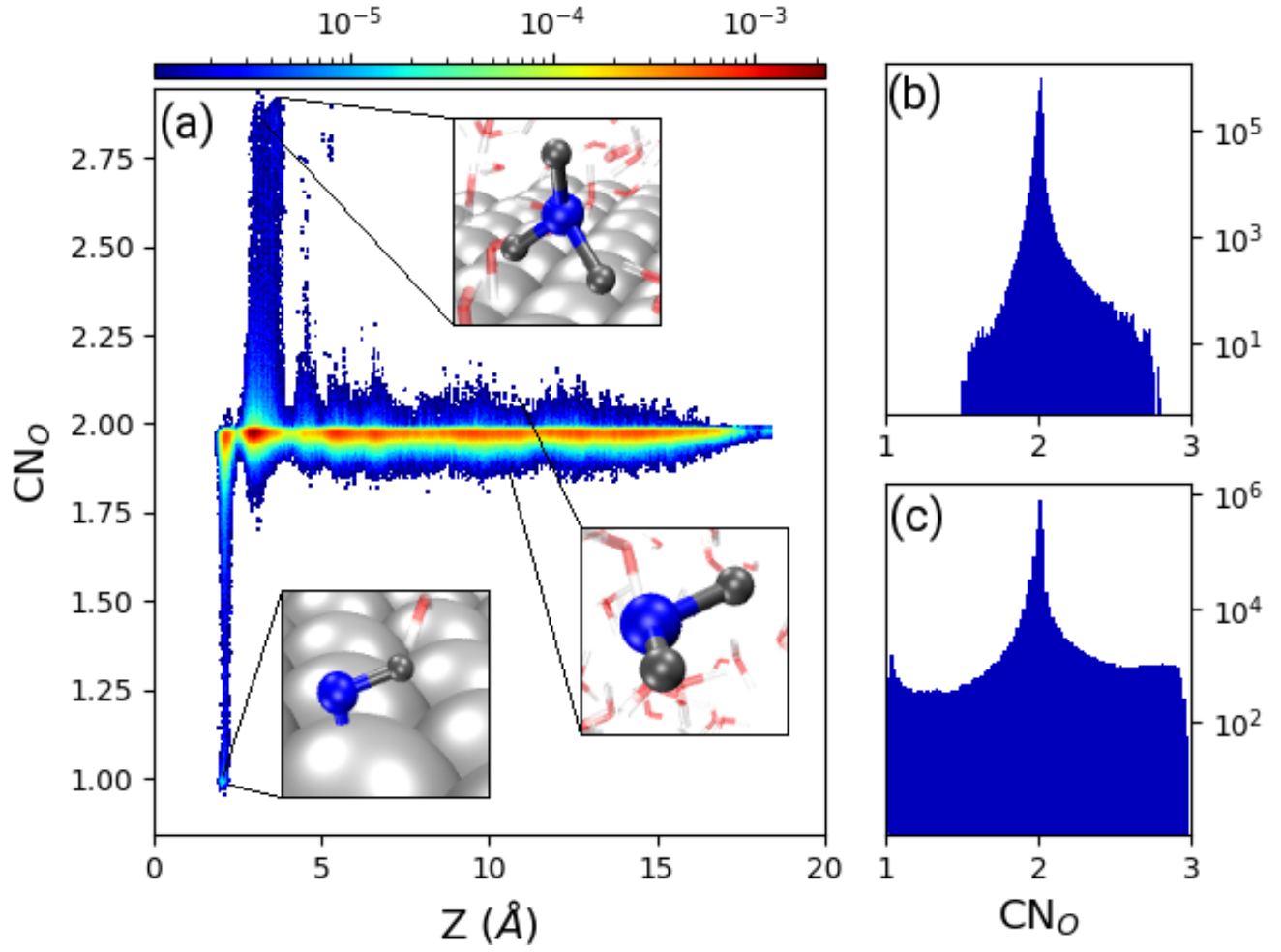

Figure 4: a) Probability distribution of $\mathrm{CN}_{O}$ as a function of O's z coordinate, calculated along the PI-AIMD trajectory of the $\mathrm{Pt} / \mathrm{H}_{2} \mathrm{O}$ system. The b) and c) panels report the effective counting for each value of $\mathrm{CN}_{O}$ within the bulk and restricted to the contact double layer, respectively. 
enhances this behaviour, since it enlarges the sampled space of configurations by favouring the delocalisation of lighter species.

The quantum sampling provided by the PI-AIMD trajectory reveals a not negligible probability for the process

$$
2 \mathrm{H}_{2} \mathrm{O}(\mathrm{DL})+\mathrm{Pt} \stackrel{k_{H}}{\rightleftharpoons} \mathrm{Pt}-\mathrm{OH}+\mathrm{H}_{3} \mathrm{O}^{+}(\mathrm{DL})
$$

From the concentration of the relevant species within the contact double layer ([Pt-OH], $\left[\mathrm{H}_{2} \mathrm{O}\right]$ and $\left[\mathrm{H}_{3} \mathrm{O}^{+}\right]$), which are obtained from the statistical analysis of $\mathrm{CN}_{O}$, we provide an estimate of the dissociation probability at equilibrium

$$
\Delta F_{\mathrm{Eq} \cdot 1}=-k_{\mathrm{B}} T \ln \frac{\left[\mathrm{Pt}-\mathrm{OH}^{-}\right]\left[\mathrm{H}_{3} \mathrm{O}^{+}\right]}{\left[\mathrm{H}_{2} \mathrm{O}\right]^{2}}=-k_{\mathrm{B}} T \ln K_{\mathrm{eq}},
$$

where $k_{\mathrm{B}}$ is the Boltzmann constant and the temperature $T$ is $300 \mathrm{~K}$. The ionized state turns out to be about $7.80 \mathrm{kcal} / \mathrm{mol}(338.4 \mathrm{meV})$ higher in energy with $p K_{\text {eq }}=5.48$. This value translates into a significantly higher self dissociation probability with respect to bulk water, where the $p K_{\text {eq }}$ has been estimated to be 13 using AIMD. ${ }^{42}$ Our investigation reveals the enhanced activity of the water molecules at the un-biased $\mathrm{Pt}(111)$, which is consistent with experimental data showing that at PZC the interfacial neutral $\mathrm{pH}$ would be $3.4{ }^{43,44}$

We have demonstrated that the sampling of the $\mathrm{OH}$ fluctuations provided NQEs are included favors the temporary formation of hydroxyl and hydronium at the Pt interface. The fact that spontaneous ionization is associated with the quantum nature of the H-nuclei is not new. Indeed, if the hydrogen behaved classically, the $\mathrm{pH}$ of the water would be $\sim 8.5(\mathrm{pKw}=17.0)$. This has been concluded by the evaluation of $\mathrm{pH}=7.4$ for deuterated bulk water. ${ }^{26,45}$ The kinetic isotope effect in electrochemical systems has also been observed, concluding that the rate constant ratio $k_{\mathrm{H}} / k_{\mathrm{D}}\left(K^{\mathrm{H} / \mathrm{D}}\right)$ for proton transfer oxygen reduction processes is 32 at low potential region $(-0.2 \mathrm{~V} \sim-0.1 \mathrm{~V}) .{ }^{32}$ In order to quantify this effect on the self dissociation at the $\mathrm{Pt}(111)$ interface, we have repeated the PI-AIMD simulation with 
heavy water (see Fig. 3(c)). The estimated free energy difference for $2 \mathrm{D}_{2} \mathrm{O} \rightarrow \mathrm{Pt}_{-} \mathrm{OD}^{-}+$ $\mathrm{D}_{3} \mathrm{O}^{+}$is $8.94 \mathrm{kcal} / \mathrm{mol}(387.81 \mathrm{meV})$, which corresponds to $p K_{\text {eq }}=6.44$. Based on the pathintegral transition state theory (Eq.S3), assuming the transmission coefficient to be equal 1 , our simulations give $K^{\mathrm{H} / \mathrm{D}}=27$ for the self-ionization at $\operatorname{Pt}(111)$ We believe that such isotopic effect would be detectable through well-designed experimental approaches ${ }^{20,32,43}$ using surface-sensitive spectroscopy. ${ }^{3,8}$

Another interesting aspect is the role of the specific metallic surface, and consequently of the strength and nature of the water-metal interaction, in determining the observed enhanced self-dissociation probability. To shed light on this aspect, we applied the same approach to consider the water $/ \mathrm{Au}(111)$ interface. (see Fig.3(d)) In this case we do not observe the same enhancement of self-ionization by including the NQEs, as demonstrated by the fact that $N_{\text {ion }}$ is nearly zero all the time.

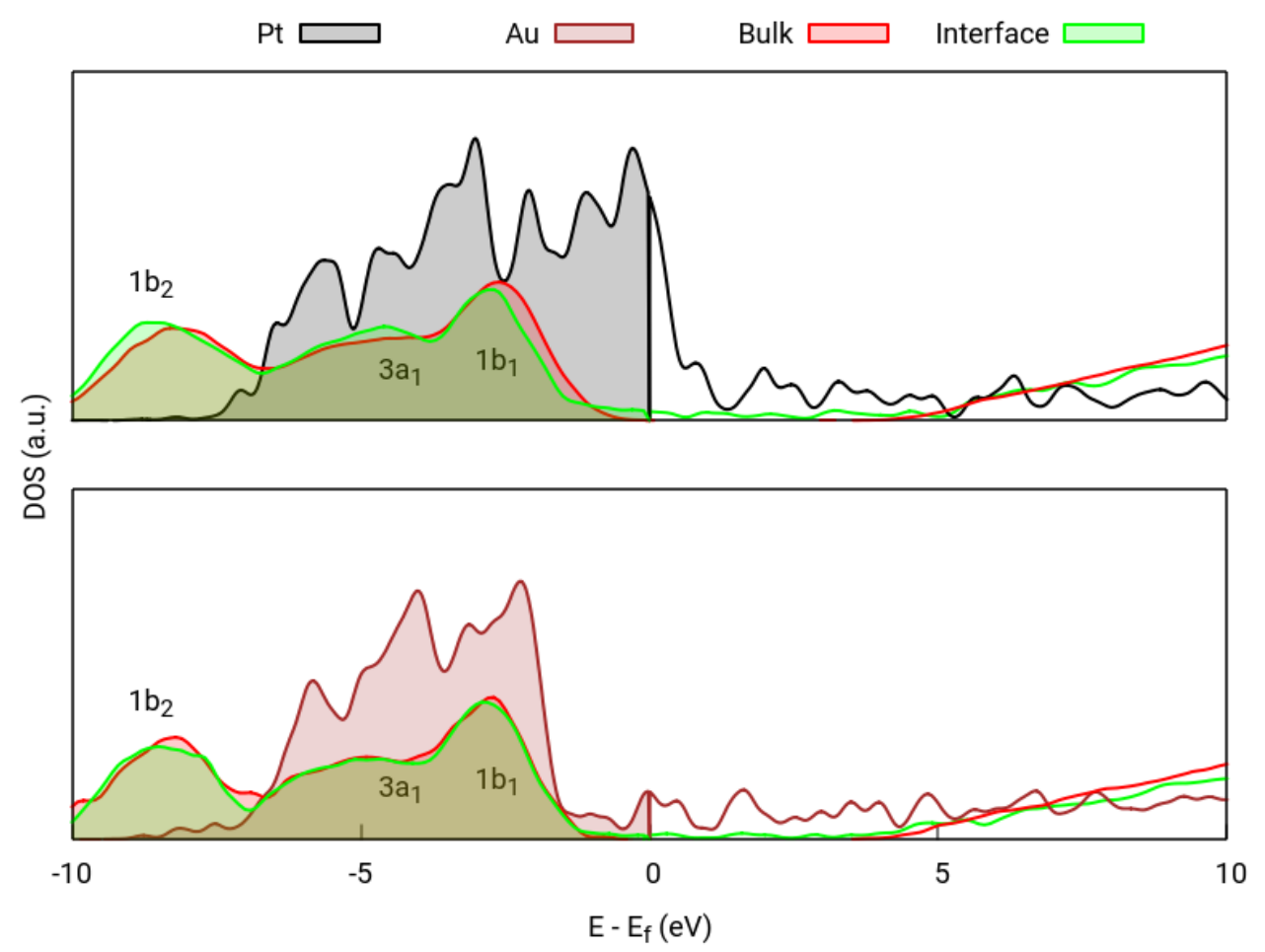

Figure 5: Projected density of states as obtained by averaging over whole PIMD trajectories. The snapshots are at time intervals of 1 ps. top: PDOS at the Pt(111) interface. bottom: PDOS at $\mathrm{Au}(111)$. The DOS of water at interface are shaded in green, bulk in red. The d-band of metal are in gray $(\mathrm{Pt})$ and brown $(\mathrm{Au})$ 
The different behavior at the $\mathrm{Pt}$ and $\mathrm{Au}$ surfaces is determined by the specific watermetal interaction. The weaker adsorption of water at $\mathrm{Au}(111)$ is also evinced by the structure of the contact water layer. The density profile reported in Fig.S4 shows a more compact slab on gold, where the contact layer appears as one single very broad peak. The density within this region is still higher than in the bulk, which determines a stronger but frustrated hydrogen bond network. However, water does not covalently bind at atop sites and the molecular orientation with respect to the surface normal is less well defined compared to what observed on $\mathrm{Pt}(111)$ (see Fig.S6). Indeed, the electric field across the interface is weaker. (additional structural analysis is provided in the Supporting Information). The projected density of states computed for the atoms at the interface confirms this picture. The difference in interaction strength can be associated to the position of the d-band center with respect to the Fermi level. On $\mathrm{Pt}(111)$ there is a high density of d-states at the Fermi energy, where also contributions from the water molecules within the contact bi-layer are present. This suggests the hybridization of the molecular orbitals of the covalently bound waters, associated to the charge transfer towards the metal, as indicated by the partial depletion of the $1 b_{1}$ band. The center of the $\mathrm{Au}(111) \mathrm{d}$-band is at $-3.91 \mathrm{eV}$, i.e., too deep to promote the hybridisation. Indeed, the distribution of the molecular states of the intrfacial water molecules is hardly modified by the interaction with the metal. This observation is in agreement with previous investigations of the metal/water interactions. ${ }^{21}$

In conclusion, by using state-of-the-art path-integral ab-initio molecular dynamics, we have shown that NQEs play a decisive role in the ionization of water at the Pt interface. This study provides new physical insight on proton hopping at aqueous metal electrode interface under ambient condition. In particular, from our results an unexpected picture of the Ptwater interface emerges, as it is generally assumed that water does not dissociate when no bias potential is applied. We instead observe that the formation of hydronium and adsorbed hydroxyl at the interface, though rare, is plausible and reversible. From the statistical analysis of the trajectories we can extract the average concentration of the adsorbed species 
and of the dissociated molecules. This provides an estimate of the equilibrium dissociation constant, indicating an increased acidity of water within the contact bi-layer interacting with $\mathrm{Pt}(111)$, which is revealed by taking into accout the quantum nature of the protons dynamics. 
Computational details All molecular dynamics simulations have been performed using the canonical ensemble at 300K. The classical AIMD is performed using the second generation Car-Parrinello dynamics ${ }^{34}$ which has been proved to be efficient and accurate for metalliquid interface. ${ }^{22}$ For PI-AIMD the PIGLET method ${ }^{28}$ implemented in I-PI ${ }^{35}$ has been used, where $\mathrm{CP} 2 \mathrm{~K}^{46}$ serves as DFT solver. Path-integral simulations were performed using 6 beads per atoms. ${ }^{28}$

Collective variables The number of ions $\left(\mathrm{N}_{\text {ion }}\right)$ defined in terms of the coordination of each oxygen to the closest $\mathrm{H}$ atoms (bonded) $\left(\mathrm{CN}_{\mathrm{O}}\right)$ has been used to evaluate the dissociation probability within the whole water slab. The analytical expressions of these quantities are

$$
\mathrm{CN}_{\mathrm{O}_{I}}(r)=\sum_{J=1}^{N_{\mathrm{H}}} \frac{1-\left(\frac{\left|\mathbf{R}_{I}-\mathbf{R}_{J}\right|}{r_{0}}\right)^{16}}{1-\left(\frac{\left|\mathbf{R}_{I}-\mathbf{R}_{J}\right|}{r_{0}}\right)^{56}}, \quad \text { where } \quad r_{0}=1.32 \AA
$$

and

$$
\mathrm{N}_{\mathrm{ion}}=\sum_{I=1}^{N_{\mathrm{O}}}\left(\mathrm{CN}_{\mathrm{O}_{I}}-2\right)^{2}
$$

For an intact water molecule $\mathrm{CN}_{\mathrm{O}} \approx 2$ and consequently $\mathrm{N}_{\text {ion }} \approx 0$. On the other hand, the presence of one hydronium as well as the presence of one hydroxide would add approximately 1 to the sum in Eq. 4. The structural analysis are based on the centroid of beads if not indicated.

Acknowledgments: This work is funded by the SNF Sinergia grant and the University Research Priority Program (URPP) for solar light to chemical energy conversion (LightChEC) of the University of Zurich. The authors thank the generous allocation of computing resource from the Swiss National Supercomputing Center (CSCS) (project uzh1, s965, s966). We thank Professor Jürg Hutter for the support and discussions. Discussions with J. Richardson (ETH) are acknowledged. J.Lan also thank additional grants from GRC and CMSZH.

\section{Supplementary Information Available}

The details of DFT calculation, atomic model, Hartree potential, simulation for heavy water and water/gold, density of states and structural information at interfaces are given in Sup- 
porting Information.

Author contributions: J.L, M.I designed research; J.L. performed research; J.L., V.R., and M.I. analyzed data; and J.L., V.R., and M.I. wrote the paper.

Code availability The computer codes used in this study are available and necessary input files to generate results are provided.

Data availability Any data generated and analyzed for this study that are not included in this article and its Supplementary Information are available from the authors upon reasonable request.

\section{References}

(1) Carrasco, J.; Hodgson, A.; Michaelides, A. A molecular perspective of water at metal interfaces. Nature materials 2012, 11, 667 .

(2) Li, C.-Y.; Le, J.-B.; Wang, Y.-H.; Chen, S.; Yang, Z.-L.; Li, J.-F.; Cheng, J.; Tian, Z.-Q. In situ probing electrified interfacial water structures at atomically flat surfaces. Nature materials 2019, 1 .

(3) Velasco-Velez, J.-J.; Wu, C. H.; Pascal, T. A.; Wan, L. F.; Guo, J.; Prendergast, D.; Salmeron, M. The structure of interfacial water on gold electrodes studied by x-ray absorption spectroscopy. Science 2014, 346, 831-834.

(4) Wu, C. H.; Pascal, T. A.; Baskin, A.; Wang, H.; Fang, H.-T.; Liu, Y.-S.; Lu, Y.-H.; Guo, J.; Prendergast, D.; Salmeron, M. B. Molecular-Scale Structure of ElectrodeElectrolyte Interfaces: The Case of Platinum in Aqueous Sulfuric Acid. Journal of the American Chemical Society 2018, 140, 16237-16244.

(5) Magnussen, O. M.; Groß, A. Toward an Atomic-Scale Understanding of Electrochemical 
Interface Structure and Dynamics. Journal of the American Chemical Society 2019, 141, 4777-4790.

(6) Hodgson, A.; Haq, S. Water adsorption and the wetting of metal surfaces. Surface science reports 2009, 64, 381-451.

(7) Van der Niet, M. J.; Garcia-Araez, N.; Hernández, J.; Feliu, J. M.; Koper, M. T. Water dissociation on well-defined platinum surfaces: The electrochemical perspective. Catalysis today 2013, 202, 105-113.

(8) Dong, J.-C.; Zhang, X.-G.; Briega-Martos, V.; Jin, X.; Yang, J.; Chen, S.; Yang, Z.-L.; Wu, D.-Y.; Feliu, J. M.; Williams, Z.-Q. T., Christopher T; Li, J.-F. In situ Raman spectroscopic evidence for oxygen reduction reaction intermediates at platinum singlecrystal surfaces. Nature Energy 2019, 4, 60.

(9) Meng, S.; Wang, E.; Gao, S. Water adsorption on metal surfaces: A general picture from density functional theory studies. Physical Review B 2004, 69, 195404.

(10) Meng, S.; Xu, L.; Wang, E.; Gao, S. Vibrational recognition of hydrogen-bonded water networks on a metal surface. Physical review letters 2002, 89, 176104.

(11) Ogasawara, H.; Brena, B.; Nordlund, D.; Nyberg, M.; Pelmenschikov, A.; Pettersson, L.; Nilsson, A. Structure and bonding of water on Pt (111). Physical review letters 2002, 89, 276102.

(12) Feibelman, P. J. Partial dissociation of water on Ru (0001). Science 2002, 295, 99-102.

(13) Feibelman, P. PJ Feibelman, Phys. Rev. Lett. 91, 059601 (2003). Phys. Rev. Lett. 2003, $91,059601$.

(14) Lilach, Y.; Iedema, M. J.; Cowin, J. P. Dissociation of water buried under ice on Pt (111). Physical review letters 2007, 98, 016105. 
(15) Sugimoto, T.; Aiga, N.; Otsuki, Y.; Watanabe, K.; Matsumoto, Y. Emergent high-T c ferroelectric ordering of strongly correlated and frustrated protons in a heteroepitaxial ice film. Nature Physics 2016, 12, 1063.

(16) Litman, Y.; Richardson, J. O.; Kumagai, T.; Rossi, M. Elucidating the Nuclear Quantum Dynamics of Intramolecular Double Hydrogen Transfer in Porphycene. Journal of the American Chemical Society 2019, 141, 2526-2534.

(17) Huang, Y.-F.; Kooyman, P. J.; Koper, M. T. Intermediate stages of electrochemical oxidation of single-crystalline platinum revealed by in situ Raman spectroscopy. Nature communications 2016, 7, 12440.

(18) Ledezma-Yanez, I.; Wallace, W. D. Z.; Sebastián-Pascual, P.; Climent, V.; Feliu, J. M.; Koper, M. T. Interfacial water reorganization as a pH-dependent descriptor of the hydrogen evolution rate on platinum electrodes. Nature Energy 2017, 2, 17031.

(19) Koper, M. T. Blank voltammetry of hexagonal surfaces of Pt-group metal electrodes: comparison to density functional theory calculations and ultra-high vacuum experiments on water dissociation. Electrochimica Acta 2011, 56, 10645-10651.

(20) Koper, M. T.; Ojha, K.; Arulmozhi, N.; Aranzales, D. Double layer of Pt (111)-aqueous electrolyte interface: Potential of Zero Charge and Anomalous Gouy-Chapman Screening. Angewandte Chemie International Edition 2019,

(21) Le, J.; Iannuzzi, M.; Cuesta, A.; Cheng, J. Determining potentials of zero charge of metal electrodes versus the standard hydrogen electrode from density-functional-theorybased molecular dynamics. Physical review letters 2017, 119, 016801.

(22) Lan, J.; Hutter, J.; Iannuzzi, M. First-Principles Simulations of an Aqueous CO/Pt (111) Interface. The Journal of Physical Chemistry C 2018, 122, 24068-24076. 
(23) Bellarosa, L.; García-Muelas, R.; Revilla-Lopez, G.; Lopez, N. Diversity at the WaterMetal Interface: Metal, Water Thickness, and Confinement Effects. ACS central science 2016, 2, 109-116.

(24) Sakong, S.; Groß, A. The electric double layer at metal-water interfaces revisited based on a charge polarization scheme. The Journal of chemical physics 2018, 149, 084705.

(25) Markland, T. E.; Ceriotti, M. Nuclear quantum effects enter the mainstream. Nature Reviews Chemistry 2018, 2, 0109.

(26) Ceriotti, M.; Fang, W.; Kusalik, P. G.; McKenzie, R. H.; Michaelides, A.; Morales, M. A.; Markland, T. E. Nuclear quantum effects in water and aqueous systems: Experiment, theory, and current challenges. Chemical reviews 2016, 116, 7529-7550.

(27) Li, X.-Z.; Probert, M. I.; Alavi, A.; Michaelides, A. Quantum nature of the proton in water-hydroxyl overlayers on metal surfaces. Physical review letters 2010, 104, 066102.

(28) Ceriotti, M.; Manolopoulos, D. E. Efficient first-principles calculation of the quantum kinetic energy and momentum distribution of nuclei. Physical review letters 2012, 109, 100604.

(29) Litman, Y.; Donadio, D.; Ceriotti, M.; Rossi, M. Decisive role of nuclear quantum effects on surface mediated water dissociation at finite temperature. The Journal of chemical physics 2018, 148, 102320.

(30) Marx, D.; Tuckerman, M. E.; Hutter, J.; Parrinello, M. The nature of the hydrated excess proton in water. Nature 1999, 397, 601.

(31) Bajaj, P.; Richardson, J. O.; Paesani, F. Ion-mediated hydrogen-bond rearrangement through tunnelling in the iodide-dihydrate complex. Nature chemistry 2019, 11, 367.

(32) Sakaushi, K.; Lyalin, A.; Taketsugu, T.; Uosaki, K. Quantum-to-Classical Transition 
of Proton Transfer in Potential-Induced Dioxygen Reduction. Physical review letters 2018, 121, 236001.

(33) Gillan, M. J.; Alfè, D.; Michaelides, A. Perspective: How good is DFT for water? The Journal of chemical physics 2016, 144, 130901.

(34) Kühne, T. D.; Krack, M.; Mohamed, F. R.; Parrinello, M. Efficient and accurate CarParrinello-like approach to Born-Oppenheimer molecular dynamics. Physical review letters 2007, 98, 066401.

(35) Kapil, V. et al. i-PI 2.0: A universal force engine for advanced molecular simulations. Computer Physics Communications 2019, 236, 214 - 223.

(36) Sato, N. Electrochemistry at metal and semiconductor electrodes; Elsevier, 1998.

(37) Limmer, D. T.; Willard, A. P.; Madden, P. A.; Chandler, D. Water exchange at a hydrated platinum electrode is rare and collective. The Journal of Physical Chemistry C 2015, 119, 24016-24024.

(38) Hassanali, A.; Prakash, M. K.; Eshet, H.; Parrinello, M. On the recombination of hydronium and hydroxide ions in water. Proceedings of the National Academy of Sciences 2011, 108, 20410-20415.

(39) Geissler, P. L.; Dellago, C.; Chandler, D.; Hutter, J.; Parrinello, M. Autoionization in liquid water. Science 2001, 291, 2121-2124.

(40) Saitta, A. M.; Saija, F.; Giaquinta, P. V. Ab initio molecular dynamics study of dissociation of water under an electric field. Physical review letters 2012, 108, 207801.

(41) Fumagalli, L.; Esfandiar, A.; Fabregas, R.; Hu, S.; Ares, P.; Janardanan, A.; Yang, Q.; Radha, m.; Taniguchi, T.; Watanabe, K.; Watanabe, G.; Gomila, K.; Novoselov, A.; Geim, Anomalously low dielectric constant of confined water. Science 2018, 360, 13391342. 
(42) Sprik, M. Computation of the pK of liquid water using coordination constraints. Chemical Physics 2000, 258, 139-150.

(43) Rizo, R.; Sitta, E.; Herrero, E.; Climent, V.; Feliu, J. M. Towards the understanding of the interfacial pH scale at Pt (l 11 1 1$)$ electrodes. Electrochimica Acta 2015, 162, 138-145.

(44) Martínez-Hincapié, R.; Sebastián-Pascual, P.; Climent, V.; Feliu, J. M. Exploring the interfacial neutral pH region of Pt (111) electrodes. Electrochemistry Communications 2015, 58, 62-64.

(45) Haynes, W. M. CRC handbook of chemistry and physics; CRC press, 2014.

(46) Hutter, J.; Iannuzzi, M.; Schiffmann, F.; VandeVondele, J. CP2K: atomistic simulations of condensed matter systems. Wiley Interdisciplinary Reviews: Computational Molecular Science 2014, 4, 15-25. 
TOC Graphic

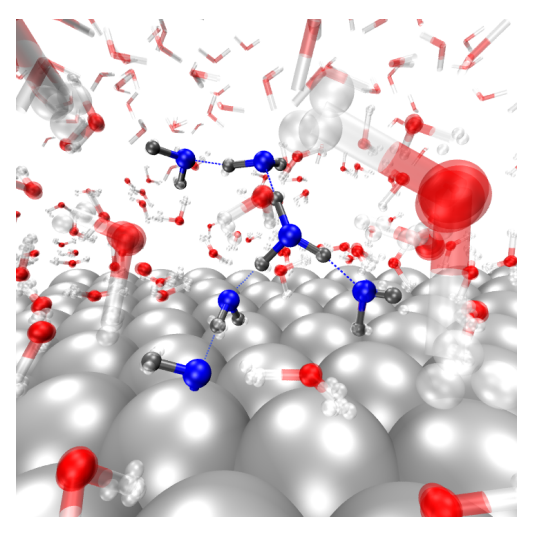

